$\xi=-1$

\title{
The Fracture Process of the Mortar Pump's Work Surfaces with Abrasive Particles
}

\author{
Roman Kaczynski $^{1}$, Oleksiyvasyliev ${ }^{2 *}$, Ievgen $_{\text {Vasyliev }}{ }^{3}$ \\ ${ }^{I}$ Bialystok University Of Technology, Poland \\ ${ }^{2}$ Poltava National Technical Yuri Kondratyuk University, Ukraine \\ ${ }^{3}$ Poltava National Technical Yuri Kondratyuk University, Ukraine \\ *Corresponding Author E-Mail: A.S.Vasiliev.76@Gmail.Com
}

\begin{abstract}
Using the example of a mortar pump with a flow-through plug the authors of the article offer a mathematical model of the fracture process of the mortar pump's work surfaces under the action of abrasive sand particles. The influence of different factors, material properties and conditions of wearing on the wear rate are analyzed in this research paper.
\end{abstract}

Keywords:. Mortar pump, abraser wear, wear rate, material performance.

\section{Introduction}

In the construction industry, when building both industrial and civic buildings, a quarter of the total labor intensivity is comprised of the works related to the use of building mortars: plastering, placing of self-leveling floors, butt joint grouting of structural units, etc. These works are carried out using plastering and troweling machines, the main part of which is the mortar pump. One of the most important problems for carrying out the stated above construction works is the provision of sufficient equipment operational life through the choice of rational forms and operating modes of mortar pumps' rubbing parts and constructional materials from which they are made.

On the basis of literary sources review $[1,2]$ it is possible to outline two ways to increase the mortar pumps reliability technological and constructive ones. The technological way of increasing the reliability of mortar pumps is to improve the manufacturing quality of the parts of the cylinder-piston group, valve units and gland boxes, drive parts, as well as to use wear-resistant materials for the manufacture of these parts, in use of modern methods of heat treatment and thermochemical treatment. In addition, the use of plasma spraying and electrodeposited coating, including hard chromium plating, enables to increase significantly the resistance to abraser wear of the coating surface of the details that are in contact with the pumped medium.

The constructive way of increasing reliability involves the creation of such a mortar pump design, which would allow to provide the most favorable solution feeding rate on condition that functional, dynamic and subgrade stresses on details and components of the mortar pump will be reduced, as well as there will be reduced the detrimental effect of abrasive particles included in the transportable mixture composition, on the parts of the cylinder-piston group and gland boxes.

The piston drive design should provide an optimal load dispatch on the parts of the cylinder-piston group, providing, on the one hand, the necessary axial operating force, and on the other hand - reducing the size of lateral components, which affects the wear rate of rubbing surfaces.

Consequently, the mortar pump components, which are exposed to the greatest destruction, are the elements that interact immediately with the building mortar.

Analyzing the main wear rate types [3, 4] of mortar pumps' rubbing surfaces, we have paid attention to the case of work surfaces destruction with a stream of harder particles. Such wear is often called erosion, or wear in the stream of floating wandering abrasive material, or hydroabrasive wear. There is a series of experimental and theoretical studies of this type of wear [5, 6], most of them are based on the idea of abrasive wear mechanism.

It is traditionally believed that in this case, along with the abrasive component, which exists at small impinging angles, there is a shock component that exists at large impinging angles. This view is based on the fact that at small impinging angles and, accordingly, at significant movement of particles on the wearing surface, the removal of material from the latter occurs as the result of microscopic cutting. But this is not obvious.

Based on the generalization of the results of multi-year research, the theory of fatigue for wear and tear [6] has been developed and experimentally confirmed (especially when it is applied to polymeric materials). In our opinion, the disadvantage of this theory is insufficient consideration of the frictional component (which was earlier called the abrasive component) of erosion wear in the context of presentations about of its mechanism. In the studies of polymeric materials wear in the abrasive particles flow, the dependence of the amount of wear on the particle velocity, we have obtained the dependence of wear rate on particle velocity, temperature, content of plasticizers, etc., but we have not managed to determine and study the mechanism of abrasive wear, that is, of microscopic cutting [5, 7].

As with the frictional interaction of surfaces, in the case under consideration, the mechanism of surface destruction may be different. If the stress or deformation that arise when the particles interact with the wearing surface do not reach the values at which the destruction occurs, then the destruction will occur after a lot of 
influences, that is, as a result of friction-contact fatigue. In the case of elastic interaction of particles with a wearing surface, the fatigue of the upper coating surface layer is close in its nature to the usual fatigue of materials during plastic deformation - this is the low-cycle fatigue $[6,8,9]$.

Further studies, accordingly, require the creation of a surface wear model under the influence of wandering abrasive particles, which would describe this process and enable to analyze it.

The purpose of this study is to create a model for the destruction process of the working surfaces of a mortar pump with a flowthrough plug, which operate in the abrasive medium, which would describe this process and allow to analyze it.

\section{Main Body}

For a better idea of the working processes, let us consider the peculiarities of the mortar pump with a flow-through plug, designed in Poltava National Technical Yuri Kondratyuk University. This technique has an extended operational life.

The significant advantage of the flow-through pump column is the reduction of flow friction (hydraulic resistance) and the so-termed "harmful" volume of the main pump working chamber, reducing the "dead" zones (blanking zones), preventing the sticking of valves and plugging during the pumping of low-slump solutions. Mortar pumps with such columns have the higher sucking capacity, hydraulic volume and volume efficiency.

The use of a flow-through plug allows for more favorable conditions for the operation of both valves in the direct solution flow in comparison with the piston. Also, the design of the flow-through piston causes the screening effect of the bulb of the pressure valve ball from the reverse flow, slows down its closure and leads to the "hanging" of the ball when pumping very dense solutions.

The plug design seems to be more attractive for the purposes of wear-resistance provision, since the formation of the outer firm surface is technologically much easier than the formation of the interior liner surface, used in the piston version.

The essential advantage of the flow-through plug is that the mortar passes through its central hole, which will allow to solve the problem of reducing the impact of hydroabrasive wear on the work surfaces of the plunger barrels, which will increase the operational life of the pump column.

In fig. 1 there is shown a scheme of a mortar pump consisting of a vertical column 1 with plunger 2 ; plunger drive, which contains the rocker arm 3, piston rod 4, connecting rod 5, pump crank 6 , pump gear box 7, electric motor 8 and V-belt transmission 9; pressure compensator of mortar 10 and pump frames. In the pump column between the two cup-type seals 13 , there is installed a flow-through plug 2 which has two cylindrical barrels, the lower and the upper ones. The cross-sectional area of the plunger's lower part is 2 times bigger than the cross-sectional area of the upper part. In the lower part of the plunger, a pressure ball valve 12 is mounted. The suction ball valve 11 and the suction adapter sleeve 14 are mounted at the bottom of the column. In the upper part of the plunger there is a spike through which the plunger is pin-connected to the rocker arm 3. The pump column and the pump drive are located on the common welded frame.

The design of the mortar pump drive due to the use of the link mechanism enables to reduce the engine load and, to a greater extent, to compensate for the impact on the movable operating element of lateral loads, which cause inequal wear.

The mortar pump works in such a way. With the help of the crank mechanism and the rocker arm, the rotational motion from the electric motor and the pump gear box is converted into the reciprocating motion of the plunger 2 . When the plunger moves upwards, the suction ball valve 11 is opened and the mortar through the suction adapter sleeve heads off to the lower part of the suction chamber 15 . At this time, the pressure ball valve 12 is closed and the mortar from the upper part of the delivery chamber 16 is squeezed out into the discharge conduit 17. The pumping rate of the mortar is determined by the difference between the cross-sectional areas of the lower and upper parts of the plunger and by its traverse speed.

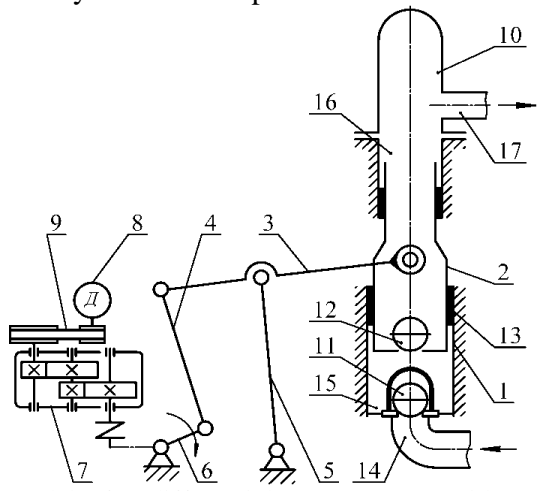

Fig. 1: The model of a differential mortar pump with a vertical flowthrough plug RNP 2-4:

1 - a vertical column; 2 - plunger; 3 - rocker arm; 4 - piston rod; 5 connecting rod; 6 - pump crank; 7 - pump gear box; 8 - electric motor; 9 - V-belt transmission; 10 - air pressure compensator; 11 - suction ball valve; 12 - pressure ball valve; 13 - cup-type seals; 14 - suction adapter sleeve; 15 - suction chamber; 16 - delivery chamber; 17 - discharge conduit.

When the plunger moves downwards, the suction ball valve 11 is closed and the mortar from the lower part of the column through the open pressure ball valve 12 enters the upper delivery chamber 16 of the column. This ensures that the first portion of the mortar mixture is fed into the pipeline.

Due to the fact that the suction ball valve 11 is closed, and the interior volume of the suction chamber 15 decreases when the plunger moves downwards due to the submersion of the plunger part into the column, the residues of the mortar are squeezed out of the column into the discharge conduit. The second portion of the mortar mixture is fed.

In the ratio of the cross-sectional area of the lower and upper parts of the plunger as $2: 1$, the pumping speed of the mortar mixture during downward motion of the plunger will be the same as during upward motion.

To reduce the pulsation of the mortar mixture, which is fed into the pipeline, an air surge dampener 10 is installed in the upper part of the column. The solution feeding $\left(2 \ldots 4 \mathrm{~m}^{3}\right.$ / hour $)$ is regulated by changing the length of the shoulders of the rocker arm 3 . This ensures the mortar pump operation with different effectiveness.

In order to improve specifications of construction equipment, new solutions are constantly being sought and modern materials are invented for the manufacture of elements and details, therefore, there is a need for a scientifically based description of the processes that occur under the action of the processed medium.

The wear rate model of work surfaces of the pump column under the action of movable wandering abrasive particles is based on the assumption that, when bumping up against the destructible surface at a certain angle, a solid particle is immersed into it and passes a certain distance along the surface $[10,11]$. When sliding, it consistently deforms several individual surface areas. If we assume that the separate surface area is a contact patch of a particle with a wearing body, then each bump of the particle will lead in general to several acts of interaction (but in different areas). Since the contact patch size changes during the interaction of particles with the surface, it will naturally be characterized by a specific specctrum, even if all the particles are spherical, of the same radius and weight, and they collide with the surface at one angle and with the same speed $[12,13]$.

For simplification, these very particles will be considered further. In addition, only the sliding of particles along the surface will be taken into account, and not possible rolling. That is, only the frictional component of wear, which is determined by the sliding of particles, is investigated in this research paper. Its shocking component is not considered in the article [14].

As is known, the wear rate at friction of firm surfaces is expressed by the ratio: 
$I=\frac{1}{A_{a}} \cdot \frac{d V}{d y}=i \frac{A_{r}}{A_{a}}$

where $I-$ the wear rate;

$V$ - the volume of material, removed from the slidepath $d y$;

$A_{a}$ - nominal friction surface;

$A_{r}-$ the actual contact area;

$i$ - specific wear.

Accordingly, specific wear is expressed by the ratio:

$i=\frac{\Delta V}{l \cdot A_{r}}=\frac{V_{\text {Д }}}{l \cdot A_{r} \cdot n}$,

where $\Delta V$ - the volume of material removed from the actual area

$A_{r}$ when moving to the mean diameter of the contact patch $l$;

$n$ - the number of cycles before destruction;

$V_{D}$ - deformed volume.

On the basis of the dependences (1) and (2) we will obtain the following:

$$
d V=\frac{V_{D}}{n \cdot l} \cdot d y .
$$

The equation (3) does not contain values $A_{r}$ and $A_{a}$ and can therefore be used to analyze the wear rate in our case.

As a wear property, let us take the dimensionless quantity of the ratio of the worn material weight to the weight of the particles that caused this wear:

$J=\frac{M_{S}}{M_{r}}$,

where $M_{S}$ - the worn material weight;

$M_{r}$ - the weight of the particles that caused this wear.

The following correspondences will also be correct:

$M_{s}=V_{s} \cdot \rho_{s}$,

where $V_{s}$ - the volume of the worn material;

$\rho_{s}$ - the density of the material that wears out;

$M_{r}=V_{r} \cdot \rho_{r}$,

where $V_{r}$ - the volume of the material causing wear;

$\rho_{r}-$ the density of particles material;

$V_{r}=V_{1} \cdot q$,

where $V_{1}$ - the volume of one particle causing wear;

$q$ - the umber of particles causing wear $V$;

$V_{1}=4 / 3 \cdot \pi \cdot R^{3}$,

where $R$ - the radius of particles.

Consequently, if we substitute the dependences (5), (6), (7) into the expression (4), we will obtain:

$$
J=\frac{V \cdot \rho_{s}}{4 / 3 \cdot \pi \cdot R^{3} \cdot \rho_{r} \cdot q} .
$$

where we can find $V$ either by integrating the expression (3) or by determining it experimentally.

We shall consider in detail the problem of elastic contact. Let the stream of solid spherical particles, which move at the speed $v_{0}$ at the angle $\alpha$ to the surface (Fig. 2), affect the flat surface of the elastic material. Each particle after the hit elastically deforms the wearable body, and slides with friction against its surface. If we assume that the normal velocity component $v_{0} \cdot \sin \alpha$ causes only elastic deformation of the material without loss, then at the rebound, this velocity component is stored in absolute magnitude
The tangential velocity component $v_{0} \cdot \cos \alpha$ is partially or completely extinguished by friction. In this case, there is frictionalcontact fatigue, which leads to the destruction and separation of the material, that is, to wear.

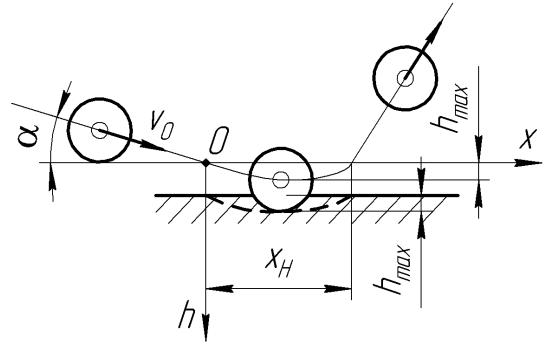

Fig. 2: Elastic interaction of a solid spherical particle with the half-space, bounded by flat surface.

Under the conditions of the independent from each other action of particles one can use the main ratio of Hertz:

$l=2 \sqrt{R \cdot h}$,

where $l$ - the mean diameter of the contact patch;

$h$ - depth of particles submergence.

$p_{r}=\frac{4}{3 \pi} \cdot \frac{E}{1-\mu^{2}} \cdot \sqrt{\frac{h}{R}}$,

where $E$ - the elasticity modulus of the wearable material;

$\mu$ - the Poisson ratio of the wearable material, as well as the previously obtained [8] ratio, which describes the fatigue curve under the friction-contact action,

$n=\left(\frac{\sigma_{0}}{k \cdot f \cdot p_{r}}\right)^{t}$.

If we substitute the relation (11) into (12), we will obtain the following:

,(13)

where $R$ - the radius of particles;

$\sigma_{0}$ - initial strength of the material;

$p_{r}$ - average pressure on the contact

$f$ - frictional coefficient of the particle sliding on the wearable material;

$t$ - coefficient of the material fatigue;

$k$ - coefficient of proportionality.

If at the moment the $q$ of particles interact with the surface, then:

$V_{D}=q \cdot V_{D_{1}}=q \cdot \pi \cdot R \cdot h^{2}$,

where $V_{D_{1}}$ - the deformed volume that occurs at each moment per one interacting particle.

If we substitute the dependances (10), (13) and (14) into the expression (3), then we will obtain the following:

$d V=\frac{q \cdot \pi \cdot R \cdot h^{2}}{\left(\frac{3}{4} \cdot \frac{\pi \cdot\left(1-\mu^{2}\right) \cdot \sigma_{0}}{E \cdot k \cdot f} \cdot\left(\frac{R}{h}\right)^{1 / 2}\right)^{t} \cdot 2(R \cdot h)^{1 / 2}} \cdot d y=$

$=q \cdot \pi \cdot R \cdot h^{2}\left(\frac{4}{3} \cdot \frac{E \cdot k \cdot f \cdot(h / R)^{1 / 2}}{\pi \cdot\left(1-\mu^{2}\right) \cdot \sigma_{0}}\right)^{t} \cdot \frac{R^{-1 / 2} \cdot h^{-1 / 2}}{2} \cdot d y=$

$=\frac{q \cdot \pi}{2} \cdot\left(\frac{4 \cdot E \cdot k \cdot f}{3 \cdot \pi \cdot \sigma_{0} \cdot\left(1-\mu^{2}\right)}\right)^{t} \cdot R^{\frac{1-t}{2}} \cdot h^{\frac{3+t}{2}} \cdot d y$.

Integrating the expression (15) along the slidepath of particles, we will get the following: 
$V=\frac{q \cdot \pi}{2} \cdot\left(\frac{4 \cdot E \cdot k \cdot f}{3 \cdot \pi \cdot \sigma_{0} \cdot\left(1-\mu^{2}\right)}\right)^{t} \cdot R^{\frac{1-t}{2}} \cdot \int_{0}^{y_{*}} h^{\frac{t+3}{2}} \cdot d y$,

where $y_{*}-$ the slidepath of a particle on the surface of the wearable body, at one collision.

Assume that, in the case under consideration, as well as in the normal collision of a hard sphere with an elastic half-space, bounded by flat surface, the maximum immersion of particles can be written down in the following form:

$h_{\max }=R \cdot\left(\frac{5 \cdot \pi \cdot \rho_{r} \cdot\left(1-\mu^{2}\right) \cdot v_{0} \cdot \sin ^{2} \alpha}{4 \cdot E}\right)^{\frac{2}{5}}$.

Let us introduce dimensionless immersion $\varepsilon=h / h_{\max }$ and dimensionless sliding $\xi=y / h_{\max }$ according to $d \varepsilon=d h / h_{\max }$ and $d \xi=d y / h_{\max }$, and use the ratio (9) taking into account the expression (16)

$$
\frac{J \cdot\left(\frac{4}{3} \cdot \pi \cdot R^{3} \cdot \rho_{r} \cdot q\right)}{\rho_{s}}=
$$$$
=\frac{q \cdot \pi}{2} \cdot\left(\frac{4 \cdot E \cdot k \cdot f}{3 \cdot \pi \cdot \sigma_{0} \cdot\left(1-\mu^{2}\right)}\right)^{t} \cdot R^{\frac{1-t}{2}} \cdot h_{\max } \frac{t+5}{2} \cdot \int_{0}^{\xi_{*}\left(\varepsilon_{*}\right)} \varepsilon^{\frac{t+3}{2}} \cdot d \xi
$$

where $\xi_{*}$ - the dimensionless slidepath of a particle from the moment of contact to the sliding cease;

$\varepsilon_{*}-$ dimensionless immersion, at which the sliding stops.

If to carry out $J$ from the expression (18), we will get the following dependence:

$$
\begin{aligned}
& J=\frac{3 \cdot \rho_{s}}{4 \cdot \pi \cdot R^{3} \cdot \rho_{r} \cdot q} \cdot \frac{q \cdot \pi}{2} \cdot\left(\frac{4 \cdot E \cdot k \cdot f}{3 \cdot \pi \cdot \sigma_{0} \cdot\left(1-\mu^{2}\right)}\right)^{t} \times \\
& \times R^{\frac{1-t}{2}} \cdot h_{\max }^{\frac{t+5}{2}} \cdot \int_{0}^{\xi_{*}\left(\varepsilon_{*}\right)} \varepsilon^{\frac{t+3}{2}} \cdot d \xi
\end{aligned}
$$

Let us simplify the expression (19):

$$
\begin{aligned}
& J=\frac{3 \cdot \rho_{s}}{8 \cdot \rho_{r} \cdot R^{3}} \cdot\left(\frac{4 \cdot E \cdot k \cdot f}{3 \cdot \pi \cdot \sigma_{0} \cdot\left(1-\mu^{2}\right)}\right)^{t} \times \\
& \times R^{\frac{1-t}{2}} \cdot h_{\max } \frac{t+5}{2} \cdot \int_{0}^{\xi_{*}\left(\varepsilon_{*}\right)} \varepsilon^{\frac{t+3}{2}} \cdot d \xi \\
& J=\frac{3 \cdot \rho_{s}}{8 \cdot \rho_{r}} \cdot\left(\frac{h_{\max }}{R}\right)^{\frac{t+5}{2}} \cdot\left(\frac{4 \cdot E \cdot k \cdot f}{3 \cdot \pi \cdot \sigma_{0} \cdot\left(1-\mu^{2}\right)}\right)^{t} \cdot \int_{0}^{\xi_{*}\left(\varepsilon_{*}\right)} \varepsilon^{\frac{t+3}{2}} \cdot d \xi .
\end{aligned}
$$

Let's substitute the ratio (17) into the expression (21):

$$
J=\frac{3 \cdot \rho_{s}}{8 \cdot \rho_{r}} \cdot\left(\frac{4 \cdot E \cdot k \cdot f}{3 \cdot \pi \cdot \sigma_{0} \cdot\left(1-\mu^{2}\right)}\right)^{t} \cdot\left(\frac{5 \cdot \pi \cdot \rho_{r} \cdot\left(1-\mu^{2}\right)}{4 \cdot E}\right)^{\frac{t+5}{5}} \times
$$

$$
\times\left(v_{0} \cdot \sin \alpha\right)^{2+\frac{2}{5} t} \cdot \int_{0}^{\xi_{*}\left(\varepsilon_{*}\right)} \varepsilon^{\frac{t+3}{2}} d \xi
$$

To simplify the analysis and perception, let us transform the expression (22) and, as a result of this, we will obtain the following:

$$
\begin{aligned}
& J=\frac{15}{8} \cdot \rho_{s} \cdot\left(5 \cdot \rho_{r}\right)^{\frac{t}{5}} \cdot\left(v_{0} \cdot \sin \alpha\right)^{2+\frac{2}{5} t} \cdot\left(\frac{k \cdot f}{3 \cdot \sigma_{0}}\right)^{t} \times \\
& \times\left(\frac{4 \cdot E}{\pi \cdot\left(1-\mu^{2}\right)}\right)^{\frac{4}{5} t-1} \cdot \int_{0}^{\xi_{s}\left(\varepsilon_{s}\right)} \varepsilon^{\frac{t+3}{2}} d \xi
\end{aligned}
$$

Let's take a closer look at plastic contact. In case of plastic contact, the particle also deforms the wearable body. The particle energy is spent both on the plastic deformation of the material, and on overcoming the adhesion forces. As in the case of rough bodies friction, in this case it is possible to use the friction ratio as an integral characteristic of energy dissipation at tangential displacement. In addition, it should be pointed out, that at the tangential displacement of the hard sphere in a plastically deformed half-space, it is influenced by the reaction, one component of which is tangential, that is, the frictional force of that decelerates the motion, and the other component tends to push a particle out from the material (Fig. 3).

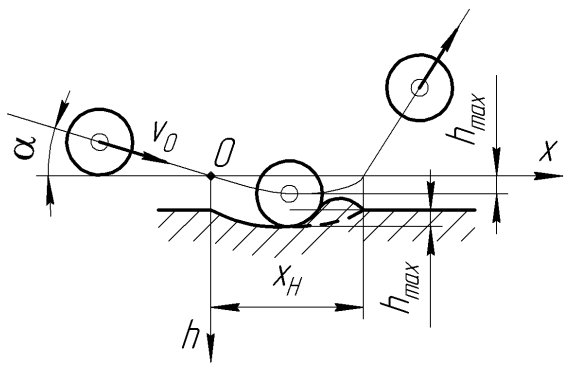

Fig. 3: Plastic interaction of a solid spherical particle with a half-space, bounded by flat surface.

During the friction of solids, both components are balanced by external forces, in the case of collision - by dynamic forces, determined by particle acceleration and mass of the particle. When having immersed as much as possible, the particle, if it continues its tangential displacement, will push out (which is the normal reaction of the material) and, of course, will continue to be inhibited by the frictional force. As soon as the particle stops its tangential displacement, there will not be its extraction from the material. Taking into account the abovementioned, for the plastic contact it is possible to leave the same approach to the calculation of wear, as to the elastic contact.

Let us use the following correspondences, known from the theory of a hard sphere sliding on a plastically deformed half-space:

$l=2 \sqrt{R \cdot h}$

$V_{D_{1}}=\pi \cdot R \cdot h^{2}$,

as well as the previously obtained ratio [8], describing the frictioncontact fatigue curve during the plastic contact,

$n=\left(e_{0} \sqrt{\frac{R}{2 h} \cdot \frac{1-k^{\prime} \cdot f}{1+k^{\prime} \cdot f}}\right)^{t}$,

where $e_{0}$ - the initial breaking strain of the material.

Let us substitute the expressions (24), (25) and (26) into the formula (3) and we will get

$$
d V=\frac{q \cdot \pi \cdot R \cdot h^{\frac{t+3}{2}}}{2 \sqrt{R \cdot h} \cdot\left(e_{0} \sqrt{\frac{R}{2 h} \cdot \frac{1-k^{\prime} \cdot f}{1+k^{\prime} \cdot f}}\right)^{t}} \cdot d y
$$

Integrating along the slidepath of the particles, we will get

$V=\frac{\pi \cdot q}{2 R^{\frac{t-1}{2}}} \cdot\left(\frac{1}{e_{0}} \sqrt{2 \cdot \frac{1+k^{\prime} \cdot f}{1-k^{\prime} \cdot f}}\right)^{t} \cdot \int_{0}^{y_{*}} h^{\frac{t+3}{2}} d y$.

Let us introduce dimensionless coordinates $\varepsilon=h / h_{\max }$ and $\xi=y / h_{\max }$ and we will transform the expression (28)

$V=\frac{\pi \cdot q \cdot h_{\max }^{\frac{t+5}{2}}}{2 R^{\frac{t-1}{2}}} \cdot\left(\frac{1}{e_{0}} \sqrt{2 \cdot \frac{1+k^{\prime} \cdot f}{1-k^{\prime} \cdot f}}\right)^{t} \cdot \int_{0}^{\varepsilon_{*}\left(\xi_{+}\right)} \varepsilon^{\frac{t+3}{2}} d \xi$.

Let us substitute the expression (29) into the equation (9) and we will get: 


$$
\begin{aligned}
& J=\frac{3 \cdot \rho_{s}}{4 \cdot \pi \cdot R^{3} \cdot \rho_{r} \cdot q} \cdot \frac{\pi \cdot q}{2} \cdot \frac{h_{\max }^{\frac{t+5}{2}}}{R^{\frac{t-1}{2}}} \times \\
& \times\left(\frac{1}{e_{0}} \sqrt{2 \cdot \frac{1+k^{\prime} \cdot f}{1-k^{\prime} \cdot f}}\right)^{t} \cdot \int_{0}^{\varepsilon_{*}\left(\xi_{*}\right)} \varepsilon^{\frac{t+3}{2}} d \xi
\end{aligned}
$$

Let's simplify the expression (30)

$$
J=\frac{3 \rho_{s}}{8 \rho_{r}} \cdot\left(\frac{h_{\max }}{R}\right)^{\frac{t+5}{2}} \cdot\left(\frac{1}{e_{0}} \sqrt{2 \cdot \frac{1+k^{\prime} \cdot f}{1-k^{\prime} \cdot f}}\right)^{t} \cdot \int_{0}^{\varepsilon_{*}\left(\xi_{*}\right)} \varepsilon^{\frac{t_{+3}}{2}} d \xi .
$$

Using the previously published [3], we will write $h_{\max }$

$$
h_{\max }=2 v_{0} \cdot R \cdot \sin \alpha \sqrt{\frac{\rho_{r}}{3 c \cdot \sigma_{s}}} .
$$

Then, based on the dependences (31) and (32), we will obtain the following:

$$
\begin{aligned}
& J=\frac{3 \rho_{s}}{8 \rho_{r}} \cdot\left(2 \cdot v_{0} \cdot \sin \alpha \sqrt{\frac{\rho_{r}}{3 \cdot c \cdot \sigma_{s}}}\right)^{\frac{t+5}{2}} \times \\
& \times\left(\frac{1}{e_{0}} \sqrt{2 \cdot \frac{1+k^{\prime} \cdot f}{1-k^{\prime} \cdot f}}\right)^{t} \cdot \int_{0}^{\varepsilon_{*}\left(\xi_{*}\right)} \varepsilon^{\frac{t_{+3}}{2}} d \xi
\end{aligned}
$$

Let us draw a simple conversion of the expression (33)

$$
\begin{aligned}
& J=\frac{\rho_{s}}{\sqrt{2}} \cdot\left(\frac{\rho_{r}}{3}\right)^{\frac{t+1}{4}} \cdot\left(\frac{v_{0} \cdot \sin \alpha}{\sqrt{c \cdot \sigma_{s}}}\right)^{\frac{t+5}{2}} \times \\
& \times\left(\frac{2}{e_{0}} \sqrt{\frac{1+k^{\prime} \cdot f}{1-k^{\prime} \cdot f}}\right)^{t} \cdot \int_{0}^{\varepsilon_{*}\left(\xi_{*}\right)} \varepsilon^{\frac{t+3}{2}} d \xi
\end{aligned}
$$

In order to determine the numerical characteristics of the wear rate of various materials, under the action of the flow of solid sand particles, it is necessary to use the obtained analytical dependences (23) and (34).

In both equations there is an integral expression, therefore, it is necessary to evaluate it:

$I=\int_{0}^{\varepsilon} \varepsilon^{\frac{t+3}{2}} \cdot d \xi$.

The dependence between $d \xi$ and $d \varepsilon$ we will take from [8]:

$d \xi=\left(f \pm \frac{\operatorname{ctg} \alpha-f}{\sqrt{1-\varepsilon^{2}}}\right) \cdot d \varepsilon$

According to the curve on Fig. 4, which shows the scheme of the particle immersion, we have the following:

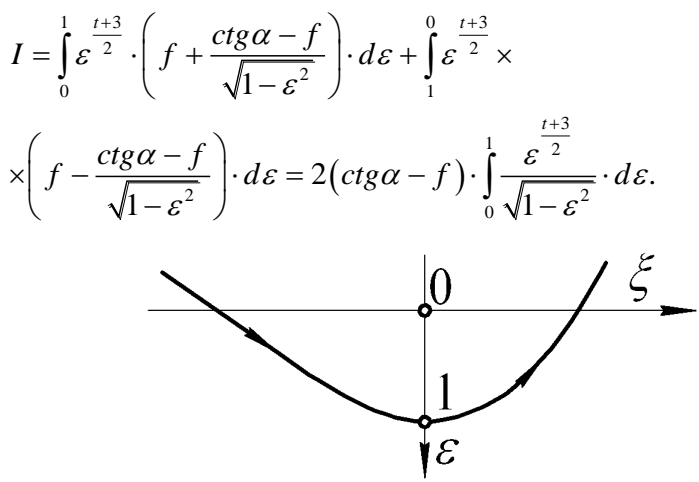

Fig. 3: The curve of a particle immersion into the material

Let us compare the wear properties $J$ for rubber and polyurethane resin. To do this, we will divide from the equations (23) and (34) a part of the expression that is substantially different for these materials, and which has equivalent values. Using the manual [8] it was established that for rubber and polyurethane resin the values $\rho_{s}, \rho_{r}, v_{0}, \sigma_{0}, E, \mu, c$ are equivalent and the friction coefficient is significantly different $f_{p}=1,79, f_{n}=1,1$. Therefore, the assessment of the wear rate of these materials should be based on these facts. Let us accept the angle at which the particle goes to the surface $\alpha=15^{\circ}, \operatorname{ctg} \alpha=\operatorname{ctg} 15^{\circ}=3,7$, and the parameters of the curve describing the friction-contact fatigue are $t \approx 2, k \approx 0,5$ Consequently, for the convenience of calculation, a part of the expressions (23) and (34), which consists of equivalent characteristics, we will denote by letters $a_{i}$.

The property of wear on elastic contact is the following:

$a_{1}=\frac{15}{8} \cdot \rho_{s} \cdot\left(5 \cdot \rho_{r}\right)^{\frac{t}{5}} \cdot\left(v_{0} \cdot \sin \alpha\right)^{2+\frac{2}{5} t} \times$

$\times\left(\frac{k}{3 \cdot \sigma_{0}}\right)^{t} \cdot\left(\frac{4 \cdot E}{\pi \cdot\left(1-\mu^{2}\right)}\right)^{\frac{4}{5} t-1}$

$J_{p}=a_{1} \cdot 1,79^{2} \cdot(3,7-1,79)=a_{1} \cdot 3,2 \cdot 1,91=a_{1} \cdot 6,112$

$J_{n}=a_{1} \cdot 1,1^{2} \cdot(3,7-1,1)=a_{1} \cdot 1,21 \cdot 2,6=a_{1} \cdot 3,146$.

The property of wear on plastic contact is the following

$$
\begin{aligned}
& a_{2}=\frac{\rho_{s}}{\sqrt{2}} \cdot\left(\frac{\rho_{r}}{3}\right)^{\frac{t+1}{4}} \cdot\left(\frac{v_{0} \cdot \sin \alpha}{\sqrt{c \cdot \sigma_{s}}}\right)^{\frac{t+5}{2}} \cdot\left(\frac{2}{e_{0}}\right)^{t}, \\
& J_{p}=a_{2} \cdot \frac{1+0,5 \cdot 1,79}{1-0,5 \cdot 1,79} \cdot(3,7-1,79)=a_{2} \cdot \frac{1,89}{0,11} \cdot 1,91=a_{2} \cdot 32,8 \\
& J_{n}=a_{2} \cdot \frac{1+0,5 \cdot 1,1}{1-0,5 \cdot 1,1} \cdot(3,7-1,1)=a_{2} \cdot \frac{1,55}{0,45} \cdot 2,6=a_{2} \cdot 8,9
\end{aligned}
$$

The obtained values allow to estimate, what material has greater resistance to wear and to what extent.

\section{Conclusion}

The analysis of the relations (23) and (34) allows to separate the influence of various factors - the material properties and conditions of wear - on the wear rate and to establish that:

1. The particles velocity $v_{0}$ affects the wear in the power index greater than two $\left(J \square v_{0}^{2+\frac{2}{5} t}\right.$ on elastic contact and $J \square v_{0}^{2+\frac{t+1}{2}}$ on plastic contact), that is, it is stronger than according to the quadratic law.

2. The particle radius does not affect the wear rate in the case when these particles are in large quantity. But the shape of a sand particle affects the pressure with which it is immersed (pressure boost increases the wear rate).

3 . The friction increase leads to a sharp increase in the wear rate.

4. Improving the characteristics affecting the durability $\left(\sigma_{0}, e_{0}, t\right)$, causes a significant reduction in wear.

5 . Increasing the elasticity modulus $E$ leads to the increased wear and tear $\sigma_{0}=$ const, while increasing the material strength $c \cdot \sigma_{s}$ (on plastic contact) - to the decrease in wear.

\section{References}

[1] Pavelieva, A., Vasyliev, I., Popov, S., \& Vasyliev, A. (2017). The analysis of running efficiency of valve units in differential mortar pump. Technology Audit and Production Reserves, 5(1(37)), 4-9. DOI: $10.15587 / 2312-8372.2017 .112351$

[2] Korobko B. (2016), Investigation of energy consumption in the course of plastering machine's work, Eastern-European Journal of Enterprise Technologies, 4/8 (82), 4 - 11. DOI: 10.15587/17294061.2016.73336. 
[3] Vinogradov V. N., Sorokin G. M., Kolokolnikov M. G. (1990), Abrazivnoe iznashivanie. M.: Mashinostroenie, 24.

[4] Surface engineering combats friction and wear. (2013). World Pumps, 2013(7-8), 8-10. DOI: 10.1016/s0262-1762(13)70205-0.

[5] Antonov, M., \& Hussainova, I. (2010). Cermets surface transformation under erosive and abrasive wear. Tribology International, 43(8), 1566-1575. DOI: 10.1016/j.triboint.2009.12.005.

[6] Rastegar, V., \& Karimi, A. (2013). Surface and Subsurface Deformation of Wear-Resistant Steels Exposed to Impact Wear. Journal of Materials Engineering and Performance, 23(3), 927-936. DOI: $10.1007 / \mathrm{s} 11665-013-0842-2$.

[7] Zhao, C. R., Jiang, J. H., \& Ke, C. (2015). Wear Testing of Principal Friction Pairs in Axial Piston Pump Based on Taguchi Design Method. Applied Mechanics and Materials, 779, 26-34. DOI: 10.4028/www.scientific.net/amm.779.26

[8] Khebdy M., Chichinadze A. V. (1989), Spravochnik po tribotekhnike. M.: Mashinostroenie.

[9] Sun, Y. (2013). Sliding wear behaviour of surface mechanical attrition treated AISI 304 stainless steel. Tribology International, 57, 67-75. DOI: 10.1016/j.triboint.2012.07.015.

[10] Braun E. D., Evdokimov Yu. A., Chichinadze A. V. (1982), Modelirovanie treniia I iznashivaniia $\mathrm{V}$ mashinakh. M.: Mashinostroenie, 191.

[11] Arulbrittoraj, A., Duraiselvam, M., \& Padmanabhan, P. (2017) Sliding wear of AISI1040 steel at different thermo chemical surface treated conditions. International Journal of Surface Science and Engineering, 11(6), 531. DOI: 10.1504/ijsurfse.2017.10010005.

[12] Korobko B., Vasyliev Ie. (2017) Test method for rheological behavior of mortar for building work. Acta mechanica et automatica, 11/3 (41), 173 - 177. DOI: 10.1515/ama-2017-0025.

[13] Fan, Y., Zhang, R. H., \& Chen, Z. B. (2013). Research on the Properties of Sea Sand Mortar. Applied Mechanics and Materials, 405408, 2871-2875. DOI: 10.4028/www.scientific.net/amm.405408.2871.

[14] Wang G.L., Ma M.L., Miao D.M., Ma H.J. (2014). Pump Ability of Concrete Mixture Improvement Based on Rich Mortar Theory Testing Method. Applied Mechanics and Materials, 472, 704 - 707. DOI: $10.4028 /$ www.scientific.net/AMM.472.704 